\title{
Three algorithms and SAS macros for estimating power and sample size for logistic models with one or more independent variables of interest in the presence of covariates
}

\author{
David Keith Williams ${ }^{1 *}$ and Zoran Bursac ${ }^{2}$
}

\begin{abstract}
Background: Commonly when designing studies, researchers propose to measure several independent variables in a regression model, a subset of which are identified as the main variables of interest while the rest are retained in a model as covariates or confounders. Power for linear regression in this setting can be calculated using SAS PROC POWER. There exists a void in estimating power for the logistic regression models in the same setting.

Methods: Currently, an approach that calculates power for only one variable of interest in the presence of other covariates for logistic regression is in common use and works well for this special case. In this paper we propose three related algorithms along with corresponding SAS macros that extend power estimation for one or more primary variables of interest in the presence of some confounders.
\end{abstract}

Results: The three proposed empirical algorithms employ likelihood ratio test to provide a user with either a power estimate for a given sample size, a quick sample size estimate for a given power, and an approximate power curve for a range of sample sizes. A user can specify odds ratios for a combination of binary, uniform and standard normal independent variables of interest, and or remaining covariates/confounders in the model, along with a correlation between variables.

Conclusions: These user friendly algorithms and macro tools are a promising solution that can fill the void for estimation of power for logistic regression when multiple independent variables are of interest, in the presence of additional covariates in the model.

Keywords: Logistic regression, Power, Sample size

\section{Introduction}

The purpose of this work is to propose and demonstrate the \%LRpowerCorr10 algorithm (and two related algorithms) which estimates power and sample size for logistic models in settings where one or more predictors are of primary interest (Additional file 1). Additionally, covariates (confounders) may be present in the model. All the potential predictors can have a specified correlation structure and may be from a mixture of different univariate distributions, namely normal, uniform, and binomial. The

\footnotetext{
* Correspondence: WilliamsDavidK@uams.edu

${ }^{1}$ Department of Biostatistics, University of Arkansas for Medical Sciences, Little Rock, AR, USA

Full list of author information is available at the end of the article
}

user inputs several conjectured attributes including sample size, correlation, and odds ratios for association between independent variables and the outcome, and the result is an estimate of power. Two other related algorithms are also described. In short, a second algorithm, \% Quickpower provides the inverse of \%LRpowerCorr10, that is, sample size for a given power. A third algorithm, \% LRpowerCorr10C provides an approximate power curve for a given range of sample sizes.

\section{Background}

The motivation for this work stems from methods that are in use to estimate power and sample size for standard linear regression models [1-4]. The MULTREG statement 
within SAS PROC POWER [1,5] allows the investigator to determine the power to detect significance for a model with set of primary predictors of interest in the presence of covariates which are included in the model, but not of primary interest. For example, suppose an investigator proposes a linear model with four total predictors X1, X2, $\mathrm{X} 3$, and $\mathrm{X} 4$ but is primarily interested in $\mathrm{X} 1$ and $\mathrm{X} 2$ while controlling for $\mathrm{X} 3$ and $\mathrm{X} 4$. To power this setting the full model would be:

$$
Y=\beta_{0}+\beta_{1} X 1+\beta_{2} X 2+\beta_{3} X 3+\beta_{4} X 4
$$

while the reduced model would be:

$$
Y=\beta_{0}+\beta_{3} X 3+\beta_{4} X 4
$$

This corresponds to testing the null hypothesis:

$$
H(0): \beta_{1}=B_{2}=0
$$

in the full model. In the best case scenario to accurately estimate power, we would like to know the difference in the R-square of the full model and R-square for the reduced model. As an illustration, the short SAS code below would return a power value of 0.864 .

\section{SAS code}

\begin{tabular}{|l|} 
proc power \\
multreg \\
model=fixed \\
alpha $=\mathbf{. 0 5}$ \\
nfullpredictors $=\mathbf{4}$ \\
ntestpredictors $=\mathbf{2}$ \\
rsqfull=0.45 \\
rsqreduced $=\mathbf{0 . 3 4}$ \\
ntotal $=\mathbf{6 0}$ \\
power $=$; \\
run;
\end{tabular}

The MULTREG statement works nicely, but requires estimates of R-squared that investigators may not know in advance. However, with some matrix algebra investigators can arrive at estimates for the R-squared for both the full and reduced models if they can provide a set of assumed correlations between each predictor $\mathrm{X}$ and $\mathrm{Y}$, along with assumed correlations among each of the variables $\mathrm{X}$. The details follow. R-squared can be expressed with the matrix expression,

$$
R^{2}=\rho_{y x} R_{x x}^{-1} \rho_{y x}^{\prime}
$$

where $\rho_{y x}$ is the $1 \mathrm{x} \mathrm{p}$ vector of simple correlations between each of the individual $\mathrm{p}$ predictors and the response variable $\mathrm{y}$, and $R_{x x}^{-1}$ is the inverse of the $\mathrm{p} \mathrm{x} \mathrm{p}$ correlation matrix among each of the predictors. Next, one can calculate R-square for the reduced model by doing the identical calculation with the removal of the predictors of interest from the rows of $\rho_{y x}$ and the rows and columns of $R_{x x}^{-1}$. An example of these calculations is as follows,

$$
R_{\text {Full }}^{2}=\left[\begin{array}{llll}
.6 & .5 & .6 & .4
\end{array}\right] *\left[\begin{array}{cccc}
1 & .6 & .7 & .5 \\
.6 & 1 & .7 & .4 \\
.7 & .7 & 1 & .5 \\
.5 & .4 & .5 & 1
\end{array}\right]^{-1} *\left[\begin{array}{l}
.6 \\
.5 \\
.6 \\
.4
\end{array}\right]=0.45
$$

where the leading row vector is the set of simple correlations of $\mathrm{Y}$ with each of the four predictors $\mathrm{X} 1, \mathrm{X} 2, \mathrm{X} 3$, and $\mathrm{X} 4$. The middle matrix is the correlation of all four predictors $\mathrm{X} 1, \mathrm{X} 2, \mathrm{X} 3, \mathrm{X} 4$ among themselves, and the last column vector is the transpose of the leading vector. The correlation values are from a particular data set and are intended for demonstration. If we are interested in investigating power for $\mathrm{X} 1$ and $\mathrm{X} 2$ while controlling for $\mathrm{X} 3$ and $\mathrm{X} 4$ we would use the calculation,

$$
R_{\text {Reduced }}^{2}=\left[\begin{array}{ll}
.6 & .4
\end{array}\right] *\left[\begin{array}{cc}
1 & .5 \\
.5 & 1
\end{array}\right] *\left[\begin{array}{l}
.6 \\
.4
\end{array}\right]=0.34
$$

in which it can be seen that the first two columns of the leading vector and the first two rows and columns of the middle matrix (which correspond to X1 and X2) have been omitted. The difference in these two calculations results in,

$$
R_{\text {Full }}^{2}-R_{\text {Reduced }}^{2}=0.45-0.34=0.11
$$

which represents another approach to providing the difference in R-squares, a quantity needed in order to calculate power for this regression model setting. A corresponding set of calculations can be done for any size set of $\mathrm{p}$ predictors with a set of predictors of interest with the compliment of this set representing the predictors that are serving for controls. It is a reasonable approach in that researchers in many instances will have some idea of the simple correlations among the response and the predictors before their study, so this approach does have its merit.

Our objective was to provide a power estimation method for logistic regression settings that work in a somewhat corresponding manner to the matrix approach above for ordinary least squares regression. Currently, all the software the authors are aware of (e.g., SAS, PASS, nQuery), estimate logistic model power of only one predictor of interest in the presence of some number of other covariates [1,4]. A well written and documented SAS macro intended for this scenario is the $\%$ PowerLog macro [6]. The \%PowerLog macro works 
nicely for this scenario but is not able to estimate power for a corresponding setting as was discussed above, that is, having more than one predictor of interest in a model controlling for other covariates. Furthermore, all these methods and software require inputs that are not always user friendly to researchers and require some initial knowledge of relationships as well as preliminary calculations. The proposed approach has the user providing the conjectured odds ratios associated with each predictor and the binary outcome, in addition to the correlations among all the predictors which seems more intuitive to many users. This approach has merit since the values of regression coefficients are equal to the natural log of the odds ratio. Demidenko et al. [7], published a similar approach using odds ratios, and currently provides an online applet (http://www.dartmouth.edu/ eugened/powersamplesize.php), however, it is applicable only to one independent variable in the presence of one confounder. Therefore our proposed methods and SAS tools extend the currently available methodology so that one can power studies for multiple independent variables of interest, in the presence of multiple covariates or confounders. In the next section we outline our algorithm to estimate power for a given sample size in this manner. It is worth noting that the SAS macros LRPowerCorr10, LRPowerCorr10C, and QuickPower that use the algorithm can accommodate up to 10 predictors, $\mathrm{X} 1-\mathrm{X} 10$. Another feature of the SAS macros is that $\mathrm{X} 1$ and $\mathrm{X} 2$ are binomial predictors, $\mathrm{X} 3-\mathrm{X} 6$ are uniform $(-3,3)$ predictors, and X7-X10 are standard normal $(0,1)$ predictors. The investigator may use any or all of these that may fit their setting.

\section{Methods}

\section{LRpowerCorr10 algorithm steps}

1. Define OR1-OR10 (the odds ratio associated with predictors X1-X10), AVEP (the average proportion of outcome $\mathrm{Y}=1$ when covariates $\mathrm{X} 1$ - X10 equal zero), and $\mathbf{P}$, the correlation matrix of the predictors.

2. Create $\mathbf{W}$, a $n \times 10$ data matrix by simulating $n$ rows of ten univariate distributions with given means and standard deviations.

3. Create $\mathbf{Z}$ by standardizing each element of $\mathbf{W}$ by subtracting the appropriate column mean and dividing by the corresponding standard deviation.

4. Define $\mathbf{P}$ the correlation matrix of the 10 predictor variables. Calculate the Cholesky decomposition of $\mathbf{P}$, that is, the matrix $\mathbf{U}$ such that $U^{\prime} U=\mathbf{P}$.

5. Calculate $\mathbf{X}=\mathbf{Z} \mathbf{U}$,

6. Multiply each element of $\mathbf{X}$ by its column's standard deviation and then add the column appropriate mean.
7. Calculate: $\operatorname{logit}=\ln (\mathrm{AVEP} /(1-\mathrm{AVEP}))+\ln (\mathrm{OR} 1) \mathrm{X} 1+$ $\ldots+\ln (\mathrm{OR} 10) \mathrm{X} 10$. Next calculate phat $=\exp (\operatorname{logit}) /$ $(1+\exp (\operatorname{logit}))$. Phat represents the probability that $\mathrm{Y}=1$ for a particular case.

8. If phat is less than or equal to a random uniform $(0,1)$ draw then $Y=1$, otherwise $Y=0$. This step is needed to convert a phat probability to an appropriate binary value in order to run PROC LOGISTIC.

9. Using SAS PROC LOGISTIC, fit the full model $y=$ X1 X2 X3 X4 X5 X6 X7 X8 X9 X10 and save the -2 log likelihood value.

10. Using SAS PROC LOGISTIC, fit the reduced model which has the predictors of interest omitted from the full model and save the -2 log likelihood value.

11. Save the difference in the full and reduced model -2 log likelihood values (likelihood ratio test; LR) [8] and determine if this value is greater or equal to the appropriate critical value. If this is the case, record this single simulation run as a 'rejection'.

12. Repeat steps $1-10 m$ times and tabulate the proportion of rejections. This proportion will be the estimate of the power for the specified scenario. Experience suggests that $\mathrm{m}=100$ is adequate to quickly evaluate scenarios. When a precise final power estimate is required, $\mathrm{m}=1000$ provides an estimate with a standard error of about 0.01 .

\section{The \%LRpowerCorr10 SAS macro}

The user must define several variables as shown in Table 1. The macro variable SAMPLESIZE corresponds to the sample size that the macro is evaluating. NSIMS is the number of simulation runs required by the user, while $\mathrm{P}$ is the correlation among all of the predictors. AVEP is the average proportion of 'yes' responses $(Y=1)$ when all the predictor values are theoretically equal to zero. OR1 through OR10 are odds ratio values associated with the predictor variables $\mathrm{X} 1-\mathrm{X} 10$. $\mathrm{X} 1$ and $\mathrm{X} 2$ are binomial variables with probability of success defined by PCX1 and PCX2. X3 through X6 are uniformly $(-3,3)$ distributed and X7 through X10 follow the standard normal distributions. The FULLMODEL macro variable has the user list the predictor variables in the full model. It should be noted that this is the literal script that is placed to the right of the equal sign in the model statement of the PROC LOGISTIC routine inside the macro, so care should be taken for accuracy. In a like manner, the REDUCEDMODEL variable is the list of predictors left in the model after the terms of interest are removed from the FULLMODEL list. ALPHA is the level of significance and DFTEST is the degrees of freedom for the likelihood ratio test [8]. This will correspond to the number of predictor terms of interest, that is, the 
Table 1 LRpowerCorr10 macro variables

\begin{tabular}{ll}
\hline SAMPLESIZE & The sample size to be evaluated \\
NSIMS & The number of simulation runs \\
P & The correlation among the predictors \\
AVEP & The average number of "1" responses in the samples \\
OR1 & The odds ratio associated with X1 (Binomial) \\
OR2 & The odds ratio associated with X2 (Binomial) \\
OR3 & The odds ratio associated with X3 ( Uni $(-3,3))$ \\
OR4 & The odds ratio associated with X4 ( Uni $(-3,3))$ \\
OR5 & The odds ratio associated with X5 ( Uni $(-3,3))$ \\
OR6 & The odds ratio associated with X6 $($ Uni $(-3,3))$ \\
OR7 & The odds ratio associated with X7 $(N(0,1))$ \\
OR8 & The odds ratio associated with X8 $(N(0,1))$ \\
OR9 & The odds ratio associated with X10 $(N(0,1))$ \\
OR10 & The predictor terms in the full model among X1-X10 \\
FULLMODEL & The predictor terms in the reduced model among \\
REDUCEDMODEL & X1-X10 \\
ALPHA & The significance level of the testing \\
DFTEST & The degrees freedom of the testing \\
PCX1 & The probability of success for X1 \\
PCX2 & The probability of success for X2 \\
\hline &
\end{tabular}

difference in the number of terms in the FULLMODEL and REDUCEDMODEL lists. Users should provide a value for OR1 through OR10. If particular predictor variables are not used in a power calculation, their corresponding OR should be set to ' 1 ' to avoid matrix algebra calculation problems. This point can be seen in practice in the provided examples.

\section{The \%QuickPower SAS macro}

The \%QuickPower macro outputs a sample size needed to achieve user specified power. The user inputs the exact same set of input variables as \%LRpowerCorr10 except SAMPLESIZE. Instead of SAMPLESIZE user inputs desired POWER, for instance 0.8. In addition user inputs number of terms in the full model (NTERMSFULL) right after the reduced model is specified. This macro allows the user to get a quick approximate idea of what sample size will be required for a given scenario. It is sometimes beneficial to run this macro first to get a ball park idea of required sample size, followed by \%LRpowerCorr 10 macro, instead of repeating simulations in order to reach desired power.

\section{The \%LRpowerCorr10C SAS macro}

The \%LRpowerCorr10C macro creates an approximate power curve for a user supplied interval of sample sizes, which can be useful for grants and papers. Instead of SAMPLESIZE or POWER, user supplies the input variable LOWER and UPPER (desired sample size range) in addition to the rest of the input set for the \%LRpowerCorr10 macro. LOWER is the minimum value on the horizontal axis of the output graph and UPPER is the maximum value.

\section{User notes and cautions}

1. To minimize numerical problems that can arise from complete separation, ensure that the product $n$ *AVEP (the product of the sample size and the average proportion where $Y=1)$, as well as the product $n^{*}(1-$ AVEP $)$, is at least 10 .

2. Caution and thought should go into the value(s) of OR and average sample proportion being evaluated for multiple logistic regression model power. If one evaluates OR3 $=2$ along with $\mathrm{AVEP}=0.1$ in the setting in which the $\mathrm{X} 3$ is from the uniform $(-3,3)$ distribution, roughly implies that the $\mathrm{P}(\mathrm{Y}=1)$ approximately doubles for each one unit increase in $\mathrm{X} 3$, which is not always reasonable. Thoughtful values of conjectured odds ratios are vital to the macro's usefulness to supply meaningful sample size and power values.

3. The \%LRpowerCorr10 macro uses the LR chi-square test statistic to evaluate power [8]. Some other power approaches use the Wald chi-square test for the power evaluation [7]. These statistics have asymptotically the same type I error and are locally equivalent, however globally they are different tests so while close, they don't always produce exactly same estimates of sample size and/or power [7]. Most statisticians would agree that the LR chi-square is generally a bit more sensitive and this implies that if one compared equivalent scenarios, it is likely that the LR chi-square approach would be slightly more powerful, but still very close.

\section{Application and results}

In the first example we demonstrate \%LRpowerCorr 10 macro by specifying a sample of 700 for a scenario with four independent covariates of interest $\mathrm{X} 1-\mathrm{X} 3$ and $\mathrm{X} 7$, two of which are binary (X1 and X2), one uniform (X3) and one standard normal (X7), with hypothesized ORs of 1.5, 1.5, 1.1 and 1.1 in bold font below, respectively. Full model also includes 4 additional covariates, X4, X8$\mathrm{X} 10$. Hypothesized correlation between variables is 0.2 , and $\mathrm{P}(\mathrm{Y}=1)=0.1$. 


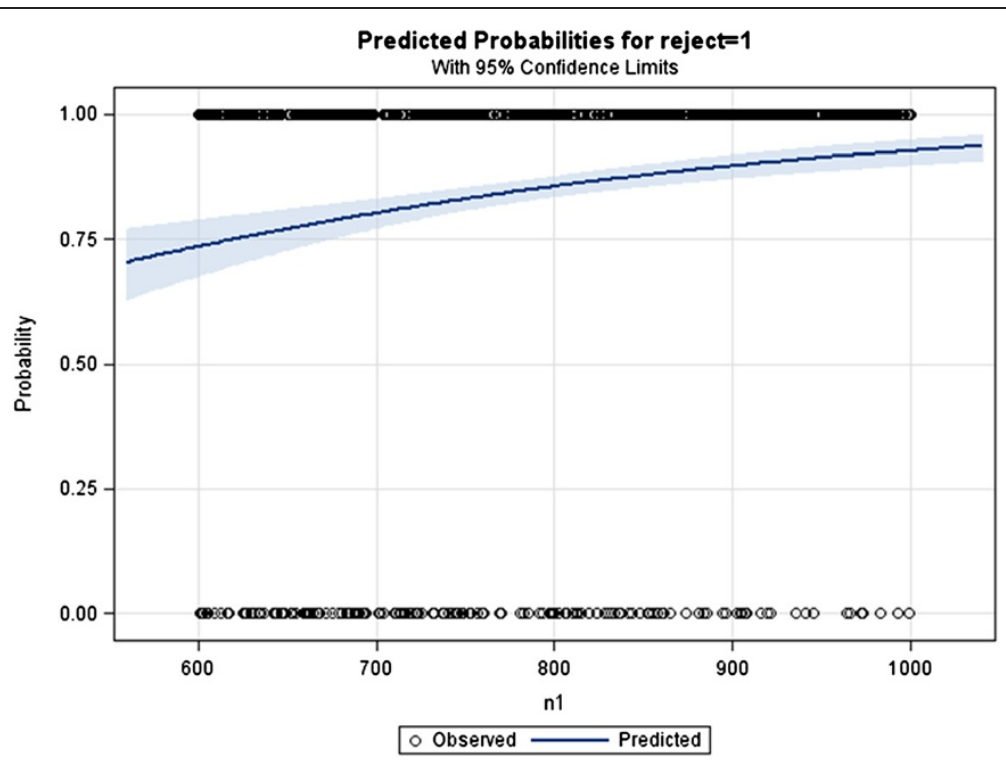

Figure $1 \%$ LRpowerCorr $10 \mathrm{C}$ macro example output.

\%LRPowerCorr10 macro example commands

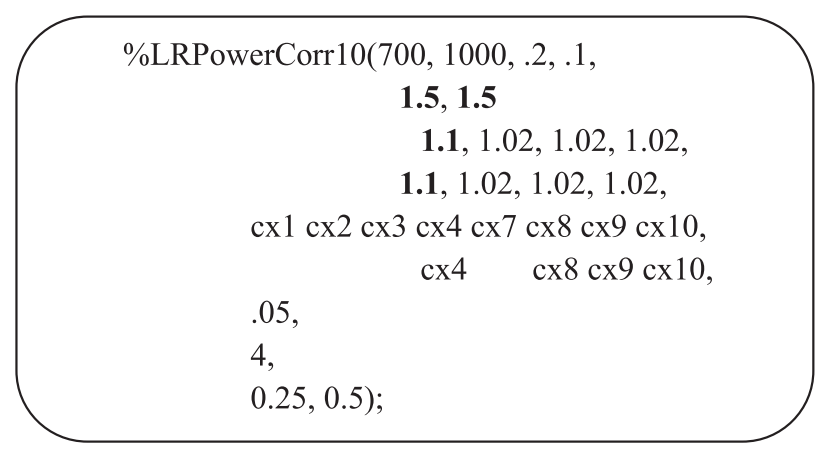

After running the algorithm described above 1000 times, macro yields the power estimate of $80 \%$ with $95 \%$ CIs ranging from $77 \%$ to $82 \%$.

\section{\%LRPowerCorr10 macro example output}

\begin{tabular}{|c|c|c|}
\hline \multicolumn{3}{|c|}{$\begin{array}{c}\text { Sample size }=700 ; \text { Simulations }=1000 ; \text { Rho }=.2 ; \mathrm{P}(\mathrm{Y}=1)=.1 \\
\mathrm{OR} 1=1.5, \mathrm{OR} 2=1.5, \mathrm{OR} 3=1.1, \mathrm{OR} 4=1.02, \mathrm{OR} 5=1.02, \mathrm{OR} 6=1.02, \\
\mathrm{OR} 7=1.1, \mathrm{OR} 8=1.02, \mathrm{OR} 9=1.02, \mathrm{OR} 10=1.02\end{array}$} \\
\hline \multicolumn{3}{|c|}{$\begin{array}{l}\text { Full Model: cx } 1 \text { cx } 2 \text { cx } 3 \text { cx } 7 \text { cx } 4 \text { cx } 8 \text { cx } 9 \text { cx } 10 \\
\text { Reduced Model: cx4 cx8 cx9 cx10 }\end{array}$} \\
\hline Power & LCL & UCL \\
\hline $80 \%$ & $77 \%$ & $82 \%$ \\
\hline
\end{tabular}

In the second example we show how to use \%Quickpower macro. The purpose of this macro is to provide a user with a quick sample size estimate for a given scenario. Below we specify the same model as in the first example with the same ORs. Instead of a sample size in this case we input the desired power, which is 0.8 in bold font. This macro also requires the number of variables in the full model which is 8 , also in bold font. Other parameters remain the same.

\section{\%QuickPower macro example commands}

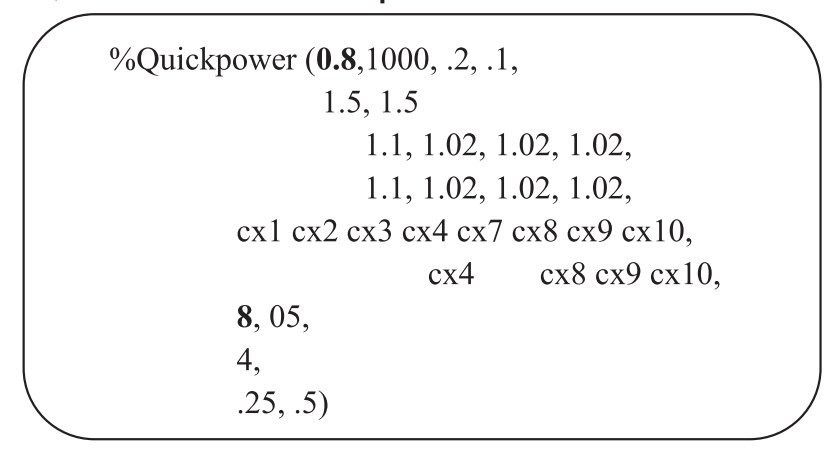

The \%Quickpower macro estimate of the sample size was 671. Within \%Quickpower macro call this estimate was inserted into \%LRpowerCorr10 macro and after running the algorithm 1000 times, macro yields the power estimate of $77 \%$ with $95 \%$ CI ranging from $74 \%$ to $80 \%$. Since the sample size of 671 appears to be slightly underpowered we could adjust it to 700 or higher as needed, and rerun the \%LRpowerCorr10 to get the power in desired range. 


\section{\%QuickPower macro example output}

$\begin{aligned} & \text { Sample size }=671 ; \text { Simulations }=1000 ; \text { Rho }=.2 ; \mathrm{P}(\mathrm{Y}=1)=.1 \\ & \text { OR1 } 1.5, \text { OR2 }=1.5, \text { OR3 }=1.1, \text { OR4 }=1.02, \text { OR5 }=1.02, \text { OR6 }=1.02, \\ & \text { OR7 }=1.1, \text { OR } 8=1.02, \text { OR9 }=1.02, \text { OR10 }=1.02\end{aligned}$
Full Model: $\mathrm{cx} 1 \mathrm{cx} 2 \mathrm{cx} 3 \mathrm{cx} 7 \mathrm{cx} 4 \mathrm{cx} 8 \mathrm{cx} 9 \mathrm{cx} 10$
Reduced Model: $\mathrm{cx} 4 \mathrm{cx} 8 \mathrm{cx} 9 \mathrm{cx} 10$
Power
LCL

In the third and final example we present the use and the results of the \%LRpowerCorr10C macro which provides an approximate power curve for the user specified range of sample sizes. Again we use the same scenario as above for consistency purposes, and we input the sample size range from 600 to 1100, in bold font below. The rest of the parameters remain the same.

\%LRPowerCorr10C macro example commands

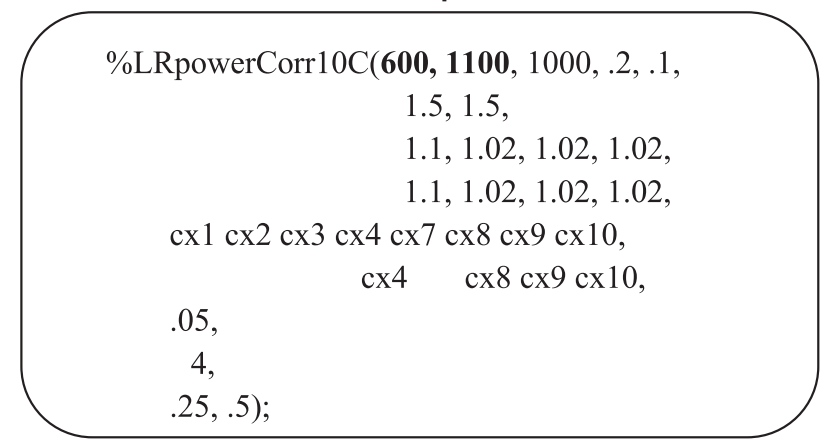

The resulting figure below shows that a sample of 600 has a power of slightly below 75\%, and as sample approaches 1100 power reaches $95 \%$. Sample size of 700 has approximate power of $80 \%$, therefore based on ones needs, desired sample size can be gauged (Figure 1).

\section{Conclusions}

The \%LRpowerCorr10 macro and the algorithm it is based on (as well as other two algorithms proposed in this paper), shows promise to fill a void for estimating power for multivariable logistic models when multiple covariates are of interest. It is able to match the approach that researchers use for multiple regression when estimating the power of a model in which one or more predictors are of interest while controlling for a number of other variables or confounders. There doesn't exist another tool on the market quite like this one, which allows us to power multiple independent covariates in the presence of additional variables in the model. Furthermore, unlike some other tools, inputs for the proposed algorithms are more intuitive in the form of odds ratios that most researchers are familiar with, and can test several possible magnitudes based on their assumptions. It allows us to specify the amount of correlation among all the predictors and attempt to match real data analysis settings that researchers commonly encounter.

\section{Additional file}

Additional file 1: Text file that contains the three SAS macros discussed in this manuscript.

Competing interests

The authors declare that they have no competing interests.

\section{Authors' contributions}

Both DKW and ZB drafted the manuscript and contributed to the development of the macros. Both authors read and approved the final manuscript.

\section{Author details}

'Department of Biostatistics, University of Arkansas for Medical Sciences, Little Rock, AR, USA. ²Division of Biostatistics and Center for Population Studies, Department of Preventive Medicine, College of Medicine, University of Tennessee Health Science Center, Memphis, TN, USA.

Received: 19 June 2014 Accepted: 27 October 2014

Published: 15 November 2014

\section{References}

1. SAS Institute Inc: SAS/STAT User's Guide, Version 9.3. Cary, NC: SAS Institute Inc; 2013.

2. Maxwell SE: Sample size and multiple regression analysis. Psychol Methods 2000, 5:434-458.

3. Hsieh FY, Block DA, Larsen MD: A simple method of sample size calculation for linear and logistic regression. Stat Med 1998, 17:1623-1634.

4. Hintze J: PASS 2012. Kaysville, UT: NCSS, LLC; 2012. www.ncss.com.

5. Castelloe JM: Sample Size Computations and Power Analysis with the SAS System. In Proceedings of the Twenty-Fifth Annual SAS Users Group International Conference, Paper 265-25. Cary, NC: SAS Institute Inc; 2000

6. Friendly M: Visualizing Categorical Data. Cary, NC: SAS Institute; 2000. http://www.math.yorku.ca/SCS/vcd/powerlog.html.

7. Demidenko E: Sample size determination for logistic regression revisited. Stat Med 2007, 26:3385-3397. http://www.dartmouth.edu/ eugened/ power-samplesize.php.

8. Shieh G: On power and sample size calculations for likelihood ratio test in generalized linear models. Biometrics 2000, 56(4):1192-1196.

doi:10.1186/1751-0473-9-24

Cite this article as: Williams and Bursac: Three algorithms and SAS macros for estimating power and sample size for logistic models with one or more independent variables of interest in the presence of covariates. Source Code for Biology and Medicine 2014 9:24.

\section{Submit your next manuscript to BioMed Central and take full advantage of:}

- Convenient online submission

- Thorough peer review

- No space constraints or color figure charges

- Immediate publication on acceptance

- Inclusion in PubMed, CAS, Scopus and Google Scholar

- Research which is freely available for redistribution 